\title{
AVANZA LA CRISIS Y EL RACISMO: RESPUESTAS DESDE ABAJO*
}

Resumen: En el actual contexto de crisis económica mundial los discursos racistas y xenófobos están cada vez más presentes en la esfera pública. Acciones y medidas que criminalizan y problematizan a la población inmigrante y a las minorías culturales están reforzando las desigualdades que ya existían. En este artículo, se argumenta que son las iniciativas provenientes de la comunidad las que están facilitando la superación de posturas que instrumentalizan la crisis para legitimar la discriminación de los sectores de la población más excluidos.

Palabras clave: racismo, crisis, segregación, diálogo, comunidad. 


\begin{tabular}{c|c}
\hline 1 & $\begin{array}{c}\text { Revista Electrónica Teoría de la Educación. } \\
\text { Educación y Cultura en la Sociedad de la Información. }\end{array}$ \\
\hline & TESI, 10(3), 2009, 198-217
\end{tabular}

\section{THE CRISIS AND RACISM ARE BOTH SPREADING: REACTIONS FROM BELOW}

Abstract: Amidst the economic crisis, racist and xenophobic discourses are increasingly present in the public sphere. Actions and measures that criminalize immigrants and cultural minorities are reinforcing previously existing inequalities. In this article, it is argued that grassroots actions are the ones are fighting against those positions which use the economic crisis to justify the advancement of discrimination towards those sectors who are more socially excluded.

Keywords: racism, crisis, segregation, community, dialogue, community

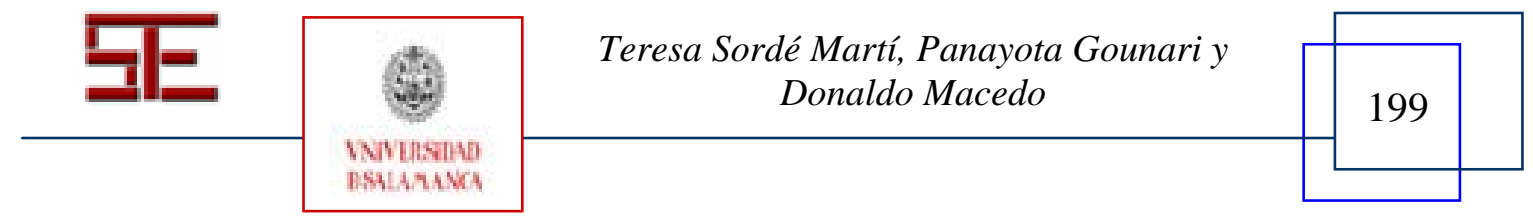




\section{AVANZA LA CRISIS Y EL RACISMO: RESPUESTAS DESDE ABAJO}

Teresa Sordé Martí.

Teresa.sorde@uab.cat

Universitat Autònoma de Barcelona

Panayota Gounari.

Panagiota.gounrai@umb.edu

Donaldo Macedo

donaldo.macedo@umb.edu

University of Massachussets, Boston.

Yo no quiero que Italia sea multiétnica, un país multicultural. Nosotros estamos orgullosos de nuestras tradiciones ${ }^{1}$.

Silvio Berlusconi

\section{1.- INTRODUCCIÓN}

Mientras los arquitectos del neoliberalismo intentan agresivamente convencer al mundo de que hemos llegado al fin de la era del racismo, Silvio Berlusconi anunciaba la aprobación de una ley de extranjería que supone un paso más a la criminalización y persecución de la población inmigrante y de minorías culturales. Desafortunadamente, las palabras de Berlusconi reiteradas de que no quiere una Italia multiétnica (Andrade, 2009) hacen eco a otras similares pronunciadas por Jean-Marie LePen en Francia, que contribuyen a contradecir el discurso que astutamente se mantiene sobre "el fin del racismo". Las expresiones xenófobas esconden esfuerzos por parte de las clases dominantes de los países desarrollados para construir socialmente algo peligrosamente siniestro: un déjá $v u$ de la ideología de la supremacía blanca que vacía nuestras sociedades de humanidad y que deja espacio para que el fascismo y el nazismo se mofen de su imagen de sociedades civilizadas. Por lo tanto, queremos mostrar cómo, en la medida que vamos entrando en el siglo veintiuno, uno de los retos más relevantes que tienen que hacer frente los educadores en las naciones desarrolladas es el espectro de una "guerra étnica y cultural”, que constituye en, nuestra opinión, un código que engendra la disposición de nuestra sociedad hacia el racismo. Central en esta guerra cultural es la fácil llamada a

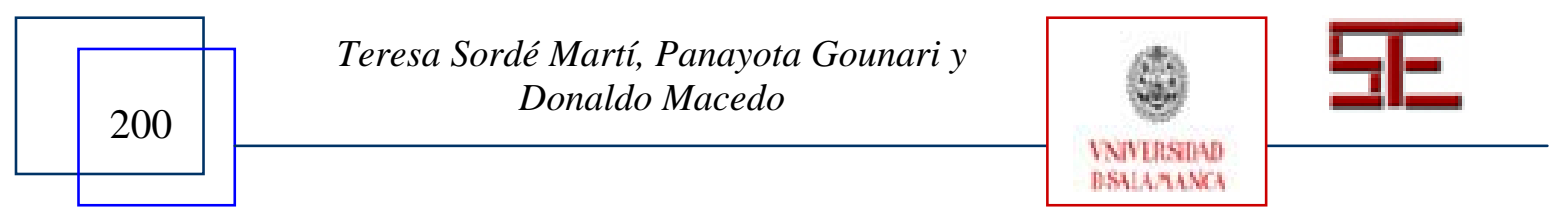




\begin{tabular}{c|c}
\hline 1 & $\begin{array}{c}\text { Revista Electrónica Teoría de la Educación. } \\
\text { Educación y Cultura en la Sociedad de la Información. }\end{array}$ \\
\hline & TESI, 10(3), 2009, 198-217
\end{tabular}

una cultura común y a la exaltación de mitos que intentan inculcarnos las creencias de la herencia de la supremacía europea. Al mismo tiempo, la ideología dominante crea otros instrumentos que degradan y devalúan otras narrativas culturales sobre la etnicidad, el lenguaje, el género, la orientación sexual y las discapacidades.

En la actual crisis económica mundial se está creando un espacio en la esfera pública donde florecen discursos racistas y xenófobos que ya existían subyacentes en la sociedad. En el seno de la Unión Europa, por ejemplo, el gobierno italiano sin ningún tipo de obstáculo ya ha aprobado medidas que nos recuerdan oscuros episodios de la historia europea. La cruzada racista y xenófoba no tan sólo ha ido en contra de la población gitana, la cual ha visto como el ministro de Interior italiano o alcaldes de diferentes ciudades apelaban a la ciudadanía a tomarse la justicia por su mano y contribuir a "eliminar a todos los campos y gitanos que hay en Italia”. Desde sus inicios, los llamados inmigrantes ilegales han sido víctimas de un proceso de criminalización que ha culminado con la aprobación de la mencionada ley. Entre otras medidas, incluye la obligación de los médicos que atienden o propietarios que alquilen casas a inmigrantes indocumentados a denunciarlos, como también autoriza la organización de patrullas ciudadanas a que "actúen” en consecuencia. La medida más controvertida ha sido la prohibición de registrar a los nacidos en el seno de "familias ilegales" en las fuentes oficiales, creando una población invisible, sin ningún tipo de derecho o protección social. La lógica es aplastante: no registrar a los bebés de familias inmigrantes, para así, contribuir a luchar contra el crimen.

Podríamos rechazar estos pronunciamientos racistas como actos de racismo extremo nacidos de la ignorancia, pero, desafortunadamente, Samuel Huntington, un profesor de Harvard que comparte unas visiones similares cuando escribe que la invasión actual de mejicanos en los Estados Unidos representa una amenaza real a la civilización americana. Uno podría argumentar que esta forma de racismo funciona más a nivel de lenguaje. Sin embargo, la relación entre sentimientos racistas y los efectos y el dolor en las vidas de las personas es directa. Esto se ejemplifica con el encarcelamiento de un padre sin documentación en una redada contra la inmigración en Texas junto con su hijo de 10 años quien pasó la noche en la cárcel antes de su puesta en libertad el día siguiente debido a las protestas generalizadas. La línea oficial es que el padre estaba siendo castigado por haber cometido el crimen de estar sin documentos de inmigración correctos y su hijo estaba en la cárcel para su propia protección ya que no había nadie fuera que lo pudiera cuidar. $\mathrm{O}$ hay el caso de una madre hondureña que la detuvieron en Texas en otra redada mientras estaba dando el pecho, siendo separadas madre e hija y pasando la segunda a disposición de trabajadores sociales. En la cárcel, ante el dolor de no poder amamantar al bebé, entregaron a la madre un sacaleches en el tercer día de encarcelamiento (Preston, 2007).

Como los ejemplos de los Estados Unidos demuestran, Italia no es el único país del mundo donde encontramos ejemplos de cómo está avanzando el racismo, manifestaciones con eslóganes racistas se están extendiendo en toda Europa. En Grecia, cinco inmi-

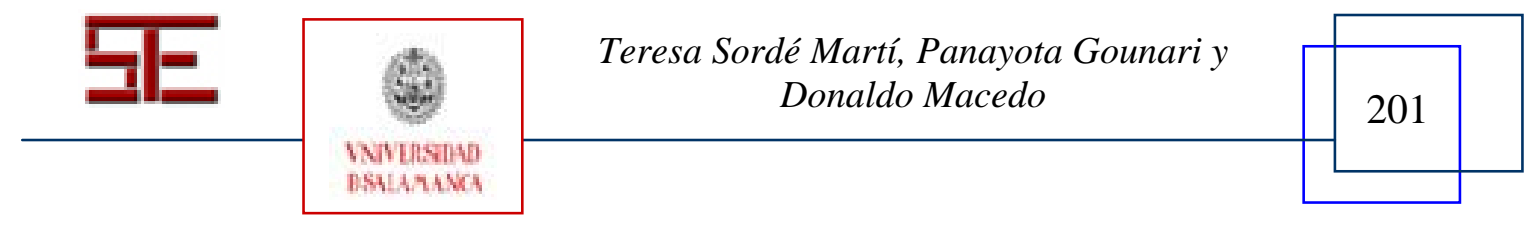


grantes resultaron heridos tras ser atacados por un grupo de extrema derecha que llevaba carteles diciendo "Extranjeros igual a crimen" o "Somos extranjeros en nuestro propio país” (BBC News, 2009) - un país donde muchas personas negarían que el racismo exista mientras las prácticas discriminatorias hacia el colectivo albano y otros grupos de inmigración más reciente se convierten en una realidad cotidiana -. En Hungría, siete personas gitanas han sido asesinadas durante este año y líderes de la comunidad denuncian que al menos ha habido más de treinta cócteles molotov lanzados contra familias gitanas (Mirga, 2009). El fenómeno de crear chivos expiatorios con los más débiles no es nuevo pero en tiempos de crisis su probabilidad crece. A medida que la crisis va afectando a más ciudadanos y ciudadanas, quedándose sin trabajo o con situaciones muy precarias, sectores conservadores se aprovechan para promover este tipo de discursos y de acciones. Por lo que hace en el ámbito educativo, los discursos de culpabilizar al alumnado inmigrante y de minorías culturales no es tampoco una realidad nueva. A pesar de múltiples investigaciones que demuestran que cualquier tipo de medida segregadora es perjudicial no tan sólo por el grupo separado sino también para el resto de estudiantes, este tipo de medida se mantiene en estos días en Europa (Flecha et al., 20062011).

Sin embargo, es imprescindible que los educadores entiendan las raíces de la segregación: la interacción entre el racismo y la gran discrepancia económica entre los llamados primeros y terceros mundos. El abismo resultante entre los países pobres y ricos se ha manifestado en patrones imprevisibles de inmigración lo cual ha agravado nuestras sociedades ya racistas. Por ejemplo, en la última década, por primera vez en la historia humana, más de cien millones de personas emigraron de una parte del planeta a otra. Con ello, este aumento exponencial en la inmigración ha llevado a un ascenso dramático en racismo y xenofobia como el caso de Italia. Adicionalmente, en Francia, el partido Front National de ultra-derechas liderado por Jean-Marie LePen, ha montado un ataque incesante sobre los inmigrantes, especialmente la población musulmana proveniente de las anteriores colonias francesas. En Alemania, ha habido un aumento importante en el número de grupos neo-nazis que han sido responsables de varios bombardeos caseros contra población griega y turca. A la vez, Turquía no se ha mantenido menos violenta contra el pueblo kurdo ya que eliminaron cientos de pueblos matando a más de treinta mil personas y condenando al resto a una vida de medio-ciudadanía en los márgenes de una existencia de guetos. En Austria, Rusia, y algunos países escandinavos los niveles de antisemitismo también están incrementando. Por su lado, Israel, alimentado por el racismo sin contener, ha aumentado el racismo violento contra la población palestina y libanesa a niveles inaceptables. En Portugal la discriminación contra la población africana de las anteriores colonias y su segregación están atestiguadas por la realidad inhumana de guetos dispersados en algunas periferias de Lisboa. Niveles similares de xenofobia también se encuentran en España donde el pueblo gitano y la población norteafricana son blancos constantes. La erupción violenta contra norteafricanos en El Ejido donde 22 personas fueron heridas constituyó un brote de racismo en un país que siempre se había afirmado como no-racista.

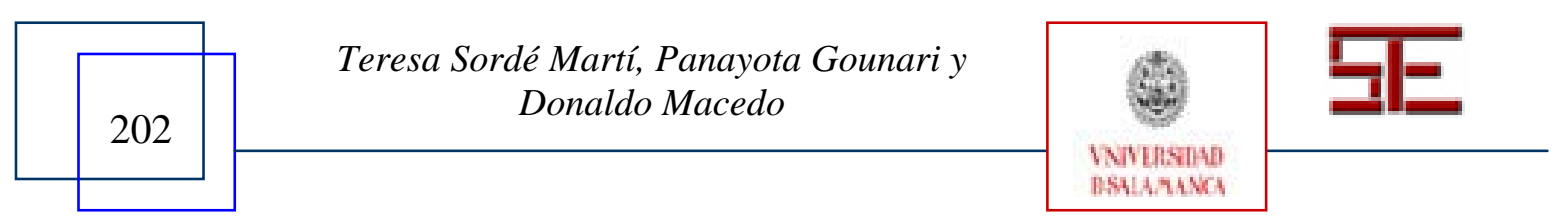




\begin{tabular}{c|c}
\hline 1 & $\begin{array}{c}\text { Revista Electrónica Teoría de la Educación. } \\
\text { Educación y Cultura en la Sociedad de la Información. }\end{array}$ \\
\hline & TESI, 10(3), 2009, 198-217
\end{tabular}

En medio de una xenofobia y un racismo globalizado que va en aumento, dudamos mucho de que la enseñanza de la tolerancia por sí sola como se hace desde varios programas multiculturales ayude a los educadores y educadoras a entender críticamente cómo las fuerzas capitalistas toman forma, moldean y mantienen la realidad cruel del racismo. También dudamos que la enseñanza de la tolerancia pueda dotar a los educadores y educadoras con las herramientas críticas para poder entender cómo se utiliza el lenguaje a menudo para construir realidades que ideológicamente esconden el racismo puro que desvalora, anula y envenena a otras identidades culturales. Aun dentro del movimiento para la educación multicultural, la mayoría de los educadores no entienden la ideología neocolonialista que informa el debate multicultural hasta el punto de que casi siempre estructuran sus argumentos dentro de una visión reduccionista de la cultura que tiene sus raíces en una herencia colonialista.

Si analizamos detenidamente la ideología que moldea y forma el debate actual sobre la educación multicultural y la polémica actual sobre la supremacía de Occidente, podemos empezar a ver y a entender que los principios ideológicos que sostienen estos debates son coherentes con las estructuras y mecanismos de una ideología colonial diseñada para devaluar el capital cultura y los valores de los colonizados. Solamente a través de un entendimiento completo de nuestra herencia colonial, podremos empezar a entender la complejidad del multiculturalismo en los países occidentales. Por ejemplo, para la mayoría de los individuos pertenecientes a culturas subordinadas en las naciones occidentales, su multiculturalismo no se caracteriza por su capacidad de tener dos culturas. Hay una diferencia radical entre cuando una persona dominante adopta una segunda cultura y cuando una persona perteneciente a una cultura subordinada lucha para adquirir y ser aceptada por la cultura dominante. Mientras la primera conlleva la adición de una segunda cultura, el último requiere la sustracción de la cultura nativa para que la persona subordinada pueda asimilarse con unos efectos psicológicos muy perjudiciales. Por lo tanto, para los grupos inmigrantes más subordinados en los países occidentales, su experiencia apunta a una forma de colonialismo interno donde sus lenguas y culturas siempre se devalúan y se sacrifican en nombre de la supremacía occidental tan exaltada.

A través del análisis de los vestigios del colonialismo que informan y moldean las manifestaciones del racismo en el mundo occidental, empezamos a comprender el papel que la historicidad ha tenido desde el principio. Por lo tanto, es necesario que miremos las maneras en que el racismo se manifiesta y se ha manifestado históricamente. A través de un marco histórico, se hace mucho más fácil adherirse a la noción de David Theo Goldberg’s que "si la raza es una concepción, entonces el racismo es una condición. O, más concretamente, donde la raza es una serie de concepciones, los racismos son una serie de condiciones"2 $(1993,100)$ y las condiciones nunca se realizan fuera de la historia. Es a través de la historia que podemos identificar mejor las estructuras que moldean y promueven "la característica dominante de la expresión racista" ${ }^{3}$ (p. 100) lo cual es, según Goldberg, la exclusión. Por lo tanto los racismos invariablemente involucran la promoción de las exclusiones y a consecuencia cualquier análisis riguroso tiene que ver los racismos como fenómenos de la exclusión articulados a diferentes niveles y los es-

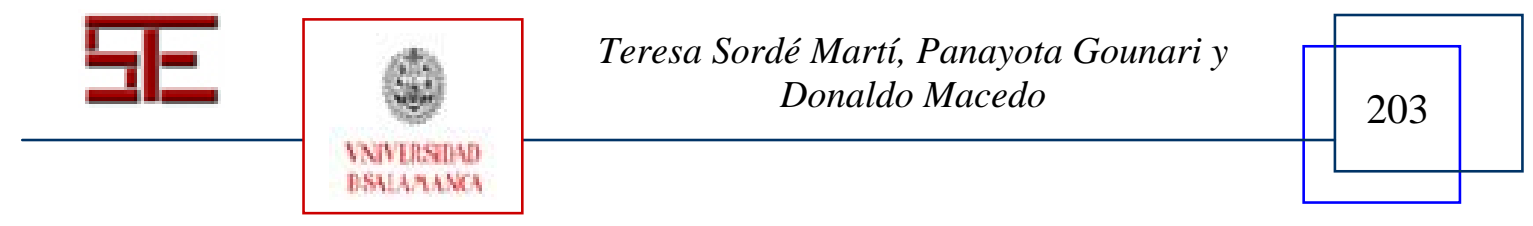


pacios que o se complementan mutuamente o entran en conflicto, construyendo una arena donde los diversos elementos geográficos, materiales, ideológicos y discursivos coexisten y se abren a la interpretación y al análisis. Nuestra discusión acerca de las causas de la segregación de la educación especial demuestra cómo la exclusión es, al final, racismo. También, las perspicacias de Goldberg son muy clarificadoras cuando afirma que "los racismos asumen sus caracteres particulares, son exacerbados, y tienen diferentes implicaciones y ramificaciones en relación a las consideraciones específicas de la constitución de clases, género, identidad nacional, y estructura política y regional”4 (p. 9). Olvidando la historia de los espacios donde el racismo ha ocupado y aún ocupa imaginativa y materialmente, estamos forzados a aceptar una noción despolitizada de la raza, mientras seguimos atrapados en un ámbito en que la "interacción política se ha prohibido" (Gilroy, 2000, 64). El "fin del racismo" inaugura un espacio despolitizado, sin debate, la lucha de los significados y las instituciones se acaban de manera simple para no despertar recuerdos peligrosos y debates incómodos. Por eso cualquier debate sobre el racismo normalmente se etiqueta como polémico, un proceso que no conlleva ningún debate ni discusión. Mientras tanto, los antagonismos raciales siempre se quedan presentes en moldear y reproducir los discursos y prácticas radicalizadas.

El racismo incluye una serie de ideologías, discursos, prácticas discursivas, instituciones y vocabularios. De todos modos, consideramos que el racismo no es simplemente una construcción ideológica, ya que, como Goldberg señala, esta visión “deja sin explicar el hecho de que las expresiones racistas pueden, a veces, definir y promover, más que meramente racionalizar, pactos sociales e instituciones" ${ }^{6}$ (p. 95). Como designación académica, abarca eruditos en múltiples áreas disciplinarias que están preocupados por la cuestión de la raza y las exclusiones resultantes. Como objeto de estudio e investigación académica, produce discursos teóricos y sociales en torno a la "raza". Esta producción de conocimiento define y da forma a nuestra interpretación del racismo, ya que también está formada por sus discursos y prácticas. El conocimiento sobre el racismo como exclusión sitúa inclusiones y exclusiones que funcionan sobre una falsa base jerárquica donde el "nosotros" está yuxtapuesto al "ellos" (del mismo modo que el mito del choque de civilizaciones) de manera que "ellos” siempre se acaba entendiendo como un valor negativo. Esta falsa dicotomía fue usada astutamente por la administración de Bush para llevar a cabo su guerra contra el terror y expandir sus ambiciones imperiales no impedidas por la oposición doméstica.

En el presente artículo, analizaremos dos ejemplos de estas medidas. En primer lugar, la sobrerrepresentación del alumnado gitano en escuelas de educación especial en países del Centro y del Este de Europa, y, en segundo lugar, medidas que en nombre de atender mejor a la diversidad legitiman prácticas que reproducen las desigualdades existentes entre grupos culturales. Finalmente, el artículo termina con ejemplos de prácticas educativas que a través de la implicación de toda la comunidad consiguen no tan sólo la inclusión de todos los grupos sino también la superación de discursos racistas y xenófobos desde abajo. La idea central del artículo es cómo se puede luchar contra el racismo y la xenofobia.

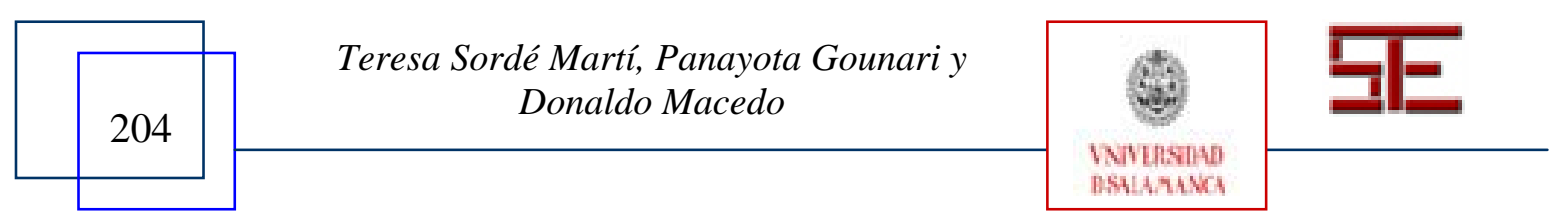




\begin{tabular}{c|c} 
Revista Electrónica Teoría de la Educación. \\
Educación y Cultura en la Sociedad de la Información.
\end{tabular}

\section{2.-ESCUELAS DE EDUCACIÓN 'ESPECIAL' PARA NIÑOS Y NIÑAS GITA- NOS}

Desde hace años, se ha denunciado la sobrerrepresentación de alumnado gitano en escuelas de educación especial. En Eslovaquia, las estadísticas oficiales indicaban que entre 1990 y 1991, dos de cada tres estudiantes en escuelas de educación especial eran gitanos (Helsinki Watch Report, 1992). Más tarde datos de la República Checa muestran que el año 1997, los niños y niñas de etnia gitana tenían 15 veces más probabilidad de estar en este tipo de escuelas que los no-gitanos (Cahn Chirico, 1999). En Hungría en el 2003, el 98\% de los estudiantes que están en las escuelas de educación especial eran de etnia gitana (ECRE, 2003). Estadísticas más recientes muestran lo difícil que es superar esta realidad.

Organizaciones de derechos humanos han denunciado las consecuencias educativas que conlleva esta discriminación, dado que la educación a la que tienen acceso estos niños y niñas es de muy escasa calidad y muy inferior a la que se recibe en el resto de escuelas. La segregación de los estudiantes gitanos en escuelas de educación especial muchas veces se esconde detrás de programas de educación bilingüe, cerrando las puertas a un futuro mejor, ya que estos niños y niñas están condenados a acceder sólo a una educación de segunda clase.

En la mayoría de los casos, no hay un procedimiento establecido para transferir a los estudiantes hacia el sistema educativo regular, de esta forma, una vez un estudiante ha sido asignado a una escuela de educación especial, en muchas ocasiones el retorno a la clase regular es casi imposible. En la República Checa y Eslovaquia, los estudiantes de educación especial una vez han acabado sólo pueden acceder a escuelas técnicas de educación compensatoria que preparan para los puestos de trabajo de más baja categoría, estas escuelas son vulgarmente llamadas "escuelas de escobas y fregona" (Danova, 2001).

Uno de los temas principales que se han denunciado como una violación de los derechos humanos de estos niños y niñas es el proceso que se sigue para asignarlos en este tipo de escuelas. Desde su diseño hasta su implementación, el test de inteligencia a partir del cual se les asigna presenta muchos problemas que se convierten en barreras muy serias para aquellos grupos, como en el caso gitano, que no controlan la lengua y cultura hegemónica. Además, se ha demostrado que el test no contempla la cultura gitana en ninguno de sus aspectos, dificultando que los niños y niñas gitanos lo pasen. También se encuentran casos de niños y niñas gitanos que han sido asignados a estas escuelas sin ni siquiera haber hecho nunca este test y sin el consentimiento de sus familias. El derecho de los padres gitanos de acceder a toda la información para poder tomar una decisión adecuada respecto a la educación de sus hijos es totalmente violado. Mientras que, por un lado, es cierto que muchos de estos niños y niñas necesitan más apoyo que el resto de sus compañeros y compañeras, a sus padres sólo se les recomienda que envíen a sus hijos a escuelas "que son más fáciles y que hacen posible estar con otros niños y

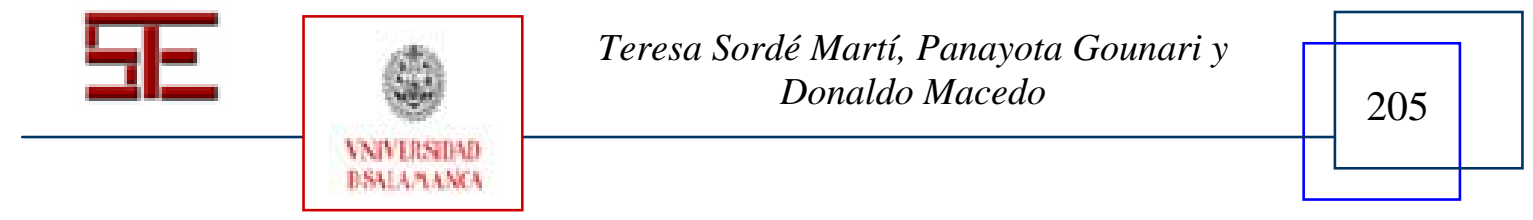


niñas gitanas que son sus amigos y familiares”. El European Committee on Romaní Emancipation (ECRE, 2003) denuncia que muchas veces estos padres deciden o autorizan el traspaso de sus hijos e hijas a estas escuelas bajo la presión o incluso el desprecio de los expertos. El silencio de estos padres puede ser interpretado como una falta de interés por su parte, pero, como se señala en investigaciones internacionales, las familias gitanas, como el resto de familias del mundo, quieren el mejor futuro para sus hijos e hijas.

Ante esta realidad existen ejemplos de familias de la República Checa y Croacia que, con la ayuda de asociaciones no gubernamentales, han denunciado su caso al Tribunal Europeo de los Derechos Humanos en Estrasburgo. En el caso de Ostrava (República Checa) el tribunal falló por primera vez en la historia de Europa, en contra de la segregación del sistema educativo checo y en defensa de los derechos civiles de las familias gitanas/os. En este caso se demostró, que en esta ciudad, la mitad de los niños y niñas gitanos estaban estudiando en escuelas de educación especial, que la mitad de estas escuelas tenían como lengua vehicular el Romanes, entre otras muchas pruebas (European Court of Human Rights, 2007). Las consecuencias educativas de esta segregación forzosa han sido extremadamente perjudiciales para el futuro de estos individuos: el currículum al que acceden está adaptado, rebajando su nivel respecto al oficial, víctimas de procesos de estigmatización y abusos psicológicos.

Hay testimonios de padres que se han movilizado en contra de las decisiones de los psicólogos de enviar a sus hijos a escuelas de educación especial, y que han tenido éxito estando ahora en escuelas regulares. A nivel organizativo, los líderes gitanos han propuesto algunas alternativas para evitar este tipo de discriminación en educación especial. Por ejemplo, la creación de escuelas de preescolar que ofrecen una base en la lengua del país antes de entrar al sistema educativo con el objetivo de prever que los niños y niñas sean asignados a escuelas especiales. Además, otras organizaciones han propuesto la transformación de escuelas de educación especial en escuelas regulares o al menos que estén dotadas de la competencia para atender las necesidades educativas de la mayoría de la población gitana. Todas ellas pasan por una implicación clara de la comunidad en este proceso como una garantía democrática, es así como se supera la segregación que no es una realidad exclusiva de Europa Central y del Este.

\section{3.- SEPARAR... ¿PARA SER MEJOR ATENDIDO? ¿PARA QUÉ?}

A pesar de que el Parlamento Europeo (2009) ha condenado en una Resolución reciente cualquier tipo de medida segregadora del alumnado inmigrante o de minorías, en muchas partes de Europa se continúan desarrollando este tipo de prácticas. En Cataluña, se han creado cuatro centros, denominados "espacios educativos de bienvenida”, destinados exclusivamente al alumnado inmigrante. Uno de ellos en Vic, la misma ciudad donde el único partido de extrema derecha ha conseguido suficientes votos para tener representantes en el ayuntamiento y donde ya se llevó a cabo un plan de redistribución del alumnado inmigrante.

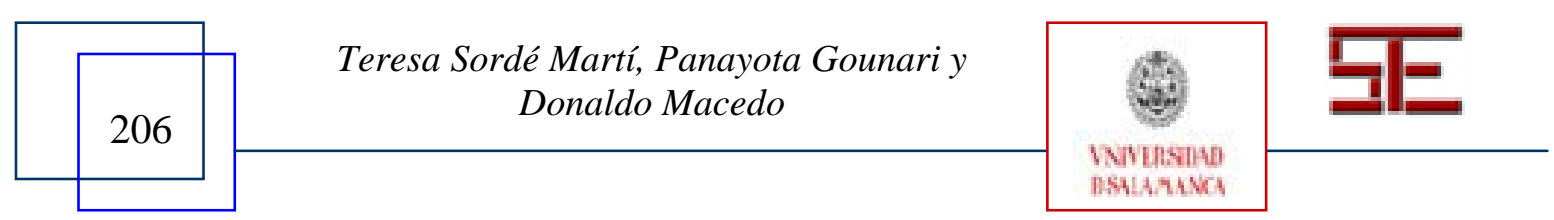




\begin{tabular}{c|c} 
Revista Electrónica Teoría de la Educación. \\
Educación y Cultura en la Sociedad de la Información.
\end{tabular}

El alumnado de minorías étnicas e inmigrante ha sido uno de los grupos más afectado por medidas que, bajo el principio de atención a la diversidad, han segregado y discriminado, enviándolos fuera del aula ordinaria. Estas medidas excluyentes no se hacen apelando a criterios étnicos o culturales sino que se justifican con explicaciones psicológicas y pedagógicas centradas en las barreras al aprendizaje que supone un bajo nivel de conocimiento previo, el desconocimiento de la lengua del país de acogida o la carencia de hábitos escolares. Sin hacer explícito que estas medidas estaban dirigidas a niños y niñas de minorías culturales o inmigrantes, en la práctica, estos espacios de exclusión se han llenado de estos chicos y chicas.

La atención a la diversidad recoge el objetivo central de la compensación de los "déficits" o carencias. A partir de este enfoque se diseña una educación para dar respuesta a los casos de niños y niñas que presentan dificultades debidas a factores sociales, económicos, geográficos, o de cualquier otro tipo, para que sigan una escolarización normalizada. En este sentido, estas medidas en lugar de compensar las desigualdades de partida y de ofrecer más a quien tiene menos, se ha convertido en una forma más de exclusión y de mantenimiento de las desigualdades (Flecha, 1994).

Estos programas segregados han tomado formas muy diferentes pero han compartido dos características: la exclusión física del chico o chica del aula ordinaria y, por tanto, la separación de su grupo-clase de referencia, y la adaptación del currículum, lo que ha supuesto la disminución de los objetivos y los contenidos de aprendizaje. El currículum que se ha diseñado para estos espacios ha priorizado aspectos vinculados con la sociabilidad por encima de aprendizajes instrumentales y de conocimientos y habilidades que son esenciales en la actual sociedad de la información. De esta forma, aunque estos espacios se diseñaron con el objetivo de atender la diversidad de alumnado y compensar sus "carencias" para ponerlos al nivel que les correspondía, por el hecho de atender la diferencia de forma desigual, han incrementado las desigualdades educativas. Si este alumnado necesitaba una aceleración de los aprendizajes para alcanzar el nivel correspondiente para su edad y curso, en estos espacios de exclusión la orientación no ha sido transformar su nivel de partida sino lo contrario, adaptar la enseñanza a las dificultades y desventajas que a diferentes niveles presentaba este alumnado. De esta forma, en lugar de aumentar los aprendizajes se han reproducido los bajos niveles y así el fracaso escolar.

A pesar de las "buenas intenciones", los enfoques de los cuales parte la educación compensatoria y la atención a la diversidad asumen que determinadas culturas o clases sociales tienen ciertos "déficits" que no les permiten acceder a un desarrollo normal en el sistema educativo. Esta racionalidad ignora las capacidades, habilidades y cualidades de estos estudiantes. Las desventajas que estos niños y niñas presentan son aceptadas y la educación que se les ofrece está adaptada a éstas en lugar de superarlas ofreciendo los mejores aprendizajes.

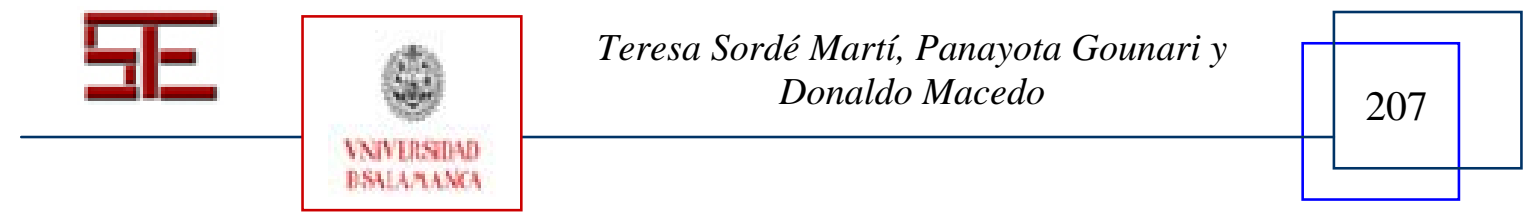


Las consecuencias a nivel personal que la segregación escolar derivada de estas asunciones ha tenido y aún hoy tiene en el alumnado gitano e inmigrante son muy negativas desde el punto de vista de mejorar su rendimiento académico, transformar sus expectativas académicas y profesionales y aumentar el sentido hacia la escuela. Todos los y las estudiantes, también aquellos que no están en estos espacios, saben que allí se aprende menos y que los y las que van tienen pocas posibilidades de tener éxito. Sabemos, por ejemplo, que en las aulas de adaptación curricular de los institutos de enseñanza secundaria se les llama "la clase del zoo", entre otros calificativos, o que al grupo de nivel más bajo cuando se hacen grupos flexibles o por niveles de habilidad en la educación primaria se les dice "el grupo de los tontos". Por todos estos motivos, estos espacios y lo que en ellos se aprende no motiva al alumnado gitano y no aumenta sus expectativas, al contrario, desmotiva y genera un autoconcepto nada positivo para aprender más.

Consecuentemente, el objetivo de que este alumnado vuelva al aula ordinaria que en principio tienen todas estas medidas segregadoras, entendidas como temporales, casi nunca se cumple. Hay muchos elementos que impiden que esto ocurra, como el fuerte etiquetaje que estos chicos y chicas reciben, las bajas expectativas que se extienden entre el profesorado, compañeros y compañeras y familias hacia sus posibilidades y la sustitución del currículum de la competencia por el currículum de la felicidad (Aubert et al., 2004). Este proceso tiene unos efectos en los mismos estudiantes que determina su futuro y que no pueden ser ignorados. Una niña gitana de catorce años decía: “A ver si con esta clase aprendo más porque soy una analfabeta de éstas. Yo sé cosas, pero no tanto como los de mi clase. Pregunta: ¿Y por qué están allí y no dentro de la clase?”. La estudiante gitana contestaba "Porque se llevan muy mal, hablan mucho... y son tontos".

En lugar de acelerar los aprendizajes y lograr un buen nivel, lo que finalmente acaba pasando en la práctica es que los aprendizajes que se hacen en estas agrupaciones de alumnado se rebajan de forma gradual, y empiezan a surgir actitudes de rebote, de rechazo hacia la escuela y los estudios, desmotivación, mal comportamiento y absentismo. Todo ello forma parte del círculo vicioso de la exclusión y el fracaso escolar del alumnado que pertenece a estos grupos. Además, lo que sucede es que en las aulas donde estos y estas alumnos/as han sido segregados siguen mostrando problemáticas. En la medida que la diversidad se sigue gestionando desde la desigualdad, las diferencias en los aprendizajes, la desmotivación y los problemas de convivencia vuelven a surgir protagonizados, esta vez, por otros alumnos que sustituyen el papel de los que ya han salido del aula.

Habría que considerar finalmente, también, los resultados que se están obteniendo con estas medidas segregadoras, ¿cuál es realmente la finalidad de estas prácticas y para qué están sirviendo, para superar el fracaso escolar o para reproducirlo? ¿Para mejorar la situación de los grupos sociales que sufren más desigualdades o para mantener y aumentar estas desigualdades? En este punto, resulta necesario promover la reflexión en torno a qué grado de cientificidad hay detrás de estas medidas segregadoras, que en otros lugares donde ya se habían aplicado ya se habían demostrado ineficaces para su-

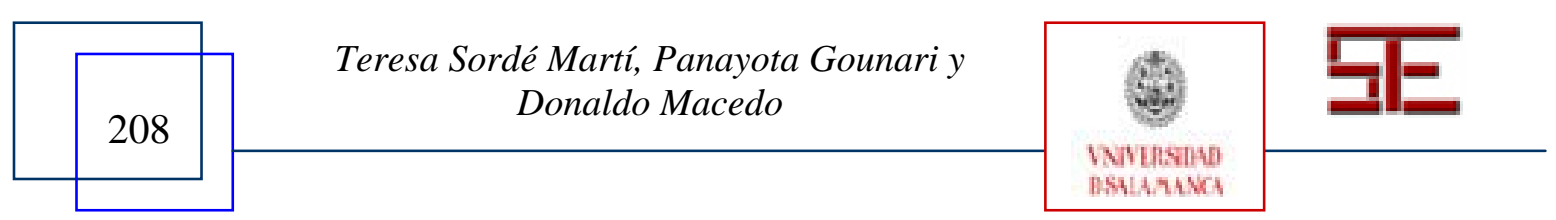




\begin{tabular}{c|c}
\hline 1 & $\begin{array}{c}\text { Revista Electrónica Teoría de la Educación. } \\
\text { Educación y Cultura en la Sociedad de la Información. }\end{array}$ \\
\hline & TESI, 10(3), 2009, 198-217
\end{tabular}

perar el fracaso escolar de los grupos que tradicionalmente han conseguido resultados más bajos en los centros escolares (Aubert et al., 2008).

Todas estas formas de segregación escolar, desde crear sistemas educativos duales, a través de las escuelas especiales o separando a los niños y niñas diferentes o conflictivos dentro de una misma escuela son instancias que refuerzan el auge del racismo. La segregación escolar es a menudo equiparada a la reproducción social, y aunque no necesariamente debe ser así, la mayoría de las veces lo es. La escuela, para niños y niñas "separados" no está siendo un espacio de transformación social que los prepare para un futuro mejor. Hay un consenso sobre que ir a escuelas o aulas separadas significa acceder a una educación desigual como también que el desarrollo personal y social mejora en la medida que tenemos la oportunidad de interaccionar con personas de culturas diferentes en espacios que fomenten y potencien el intercambio multicultural. Pero esto se dará en la medida en que en estos espacios se aseguren unos niveles de aprendizaje de máximos. Las familias y sobre todo las mujeres que están participando en escuelas que, en lugar de separar, unen los esfuerzos para que cada vez más la escuela abra las puertas a un futuro mejor para sus hijos e hijas.

\section{4.- CONTRA EL RACISMO, EL DIÁLOGO DESDE ABAJO}

Los discursos racistas y xenófobos están contestados desde las comunidades a través de su implicación directa en la educación de sus hijos e hijas. A menudo la participación de las familias gitanas en las escuelas es concebida como un elemento distorsionador, un problema y una limitación. Esta perspectiva deriva de tendencias homogeneizadoras, que se basan en enfoques que pretenden asimilar las diferencias, o de tendencias relativistas, que parten de un respeto por las diferencias que, en la adaptación a la diversidad, no sólo consiguen resultados reproductores sino que empeoran las desigualdades sociales y económicas que ya había de partida.

Por lo contrario, cada vez hay más familias gitanas que identifican la oportunidad de implicarse en las escuelas de sus hijos e hijas como una fuente de encantamiento y de encontrar sentido a sus acciones, ya que encaminan a sus hijos e hijas y a su comunidad hacia un futuro mejor. En el paso de la sociedad industrial a la sociedad de la información y fruto de los rápidos cambios producidos, la inseguridad laboral, la pérdida de los valores tradicionales, las incertidumbres en el ámbito familiar o económico, la falta de expectativas de futuro entre los jóvenes, entre otros procesos, han generado una crisis de sentido. En el ámbito escolar este desencanto también está presente y entra en estrecha relación con elementos muy concretos, como la crisis de autoridad en las aulas, la falta de expectativas en el éxito escolar del alumnado, la pérdida de los patrones y las normas tradicionales, y también con un modelo familiar cada día más cambiante.

La creación de sentido se consigue en el momento que las personas se sienten protagonistas de su realidad, deciden por sí mismas y orientan su vida hacia sus expectativas y sus sueños (Flecha, 2000). En el polo opuesto está la imposibilidad de cambio y la imposición de normas y valores que son extraños y ajenos. En este polo opuesto, también

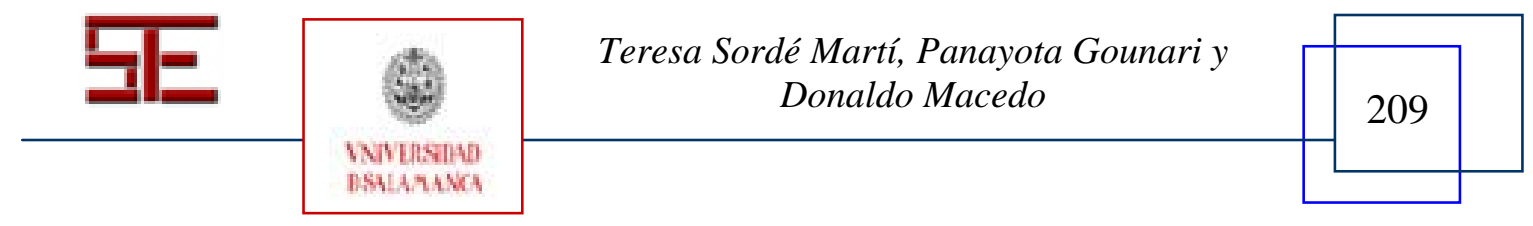


se dan situaciones en que los aprendizajes instrumentales más relevantes en la sociedad de la información no se garantizan para determinados sectores del alumnado. También es el caso de las personas de culturas minoritarias, que no se sienten reflejadas en los libros de texto, ni en la estructura temporal de la escuela y que, incluso, pueden sentirse cuestionadas por el estilo de vida que llevan.

El reencantamiento de los estudiantes de minorías étnicas en el ámbito escolar se consigue cuando escuela y comunidad educativa comparten un mismo proyecto, un mismo objetivo, y ven que su cultura es reconocida y valorada por el profesorado y sus compañeros (Elboj et al., 2002). Aunque a menudo se hace difícil encontrar este propósito común, hay uno que parece que es el más importante y que motiva más unos y otros: la educación de los niños y niñas. Claro que todo padre y toda madre quiere lo mejor para su hijo e hija y, en la sociedad de la información, lo mejor exige adquirir unos altos niveles educativos. En cuanto al profesorado, parece evidente que, como profesionales de la educación, comparten con los familiares este objetivo, aunque a veces este propósito parece que no se traduce en una práctica que lo haga posible. A menudo, lo que algunos maestros quieren para sus propios hijos e hijas no se corresponde con lo que garantizan a los niños y niñas de la escuela donde trabajan. Las bajas expectativas, la adaptación a la diversidad, el mismo desencanto ante las dificultades generadas por el fracaso escolar y los problemas de convivencia y, en definitiva, el estancamiento en una cultura de la queja que hace rebajar los niveles de exigencia y curriculares, todo hace que se pierda de vista esta meta inicial. Los niveles de aprendizaje que se alcanzan en determinadas escuelas están conectados a la implicación de las familias gitanas. A través de la participación de las familias, se generan mecanismos que fomentan un sistema más democrático y transparente que responde realmente a las necesidades de la escuela y los familiares. Se hace necesario, entonces, recuperar ese objetivo común que escuela y comunidad pueden compartir y crear sentido en las participaciones democráticas.

La implicación de las entidades de los barrios, como las asociaciones gitanas dentro de los mismos centros, es también una forma de preparar a la ciudadanía para rechazar de una forma crítica los discursos xenófobos y racistas que se van formulando. A partir de aquí, muchas personas pueden ver con diferentes ojos la escuela y las personas que trabajan, ya que hay referentes culturales que colaboran codo a codo con familia y escuela. Si estas familias y entidades encuentran un sentido en su implicación en la escuela del barrio, simplemente porque ven cómo se pueden realizar actividades conjuntamente, no sólo mejoran los rendimientos escolares de sus hijos e hijas sino los de todos los vecinos y vecinas del barrio.

Bajo el principio de la igualdad de diferencias ninguna persona, sea de la etnia que sea, se siente cuestionada ni asimilada. Tampoco ve rebajados los objetivos educativos hacia su comunidad, sino que día a día comprueba que tiene más acceso a la igualdad, no sólo de oportunidades, sino también de resultados, sin que por ello deba renunciar a su identidad cultural. Bajo el principio de la igualdad de diferencias, la diversidad cultural se entiende como un elemento plenamente enriquecedor que ofrece la oportunidad de

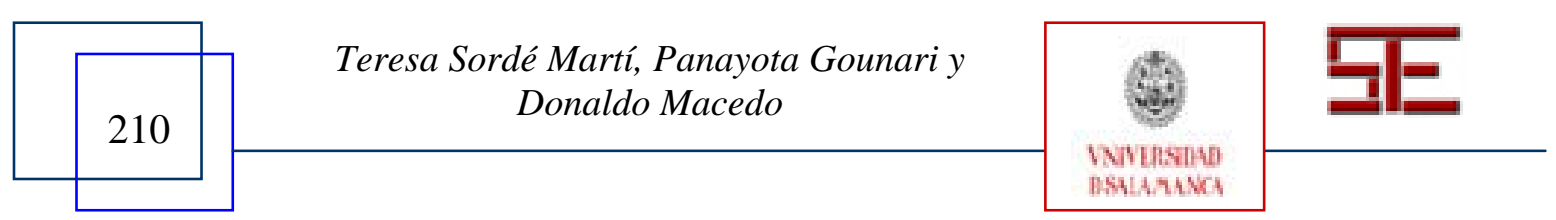




\begin{tabular}{c|c} 
Revista Electrónica Teoría de la Educación. \\
Educación y Cultura en la Sociedad de la Información.
\end{tabular}

construir una escuela más multicultural y, por tanto, más plural, democrática e inclusiva de todas las voces, en que se da la oportunidad de que los conocimientos comunitarios se multipliquen mientras que se construyen nuevos valores interculturales. Desde este punto de vista, todas las personas tienen el mismo derecho a ser diferentes. Los centros educativos consiguen trabajar la diversidad desde concepciones democráticas (Aubert et al., 2008).

Si se asegura un espacio donde todas las personas pueden ser diferentes, y donde todas las culturas están reconocidas en igualdad de condiciones, se potenciará, sin duda, la participación de las familias. En las escuelas donde los familiares se sienten como en casa y han hecho suyos los diferentes espacios como la sala donde toman café o donde hacen reuniones, en que no necesitan preguntarse continuamente donde pueden encontrar material para tomar apuntes en una reunión o hacer fotocopias de las actas de la asociación de familiares, se crea un clima en que las familias se sienten cómodas, lo que atrae más su participación.

El reconocimiento de todas las diferencias desde un principio de igualdad sólo se consigue a través de un diálogo democrático e igualitario que ha resultado ser la clave en todas las escuelas y experiencias en que se ha conseguido fomentar de manera amplia la participación. Este principio parte de las aportaciones teóricas de autores como Freire (1997) o Habermas (1984, 1987), en todos los casos, aparece como requisito necesario para la inclusión de todas las voces en el espacio escolar, familiar, laboral o social.

Generalmente, y de manera tradicional, las relaciones entre familia y escuela se han basado en relaciones de poder. Además, la falta de diálogo que suele haber en estas relaciones genera un escaso intercambio de impresiones sobre los contenidos e intereses educativos, lo que promueve la creación de prejuicios. Del mismo modo, la falta de diálogo entre familias de diferentes culturas puede conllevar conflictos y malentendidos, situación que cambia radicalmente cuando se comparten objetivos y motivaciones. Por un lado hay una clara preocupación y un gran interés desde las escuelas para fomentar la participación e implicación de los agentes sociales de su entorno dentro de los centros, pero a la vez también hay resistencias a esta apertura de la escuela a la comunidad derivadas de una cierta desconfianza y temor que se distorsione el funcionamiento del centro. Es en este punto cuando los discursos racistas o xenófobos se hacen presentes en el día a día. Sin una clara apertura se hace imposible la entrada de familiares en las escuelas y, por tanto, se dificulta el proceso de romper barreras, como el miedo a construir una institución más democrática y multicultural favorece la cultura de la queja, que genera actitudes muy paralizadoras y conservadoras.

Si uno de los aspectos que más reticencia genera a las familias la hora de ir a la escuela es el miedo de recibir comentarios negativos sobre sus hijos, hay que hacer un cambio en este sentido. La primera vez las experiencias negativas previas generarán tensiones, pero a medida que su visita a la escuela signifique algo más que referencias negativas, estas tensiones van disipándose. Del mismo modo, y tal como ya hemos comentado, es

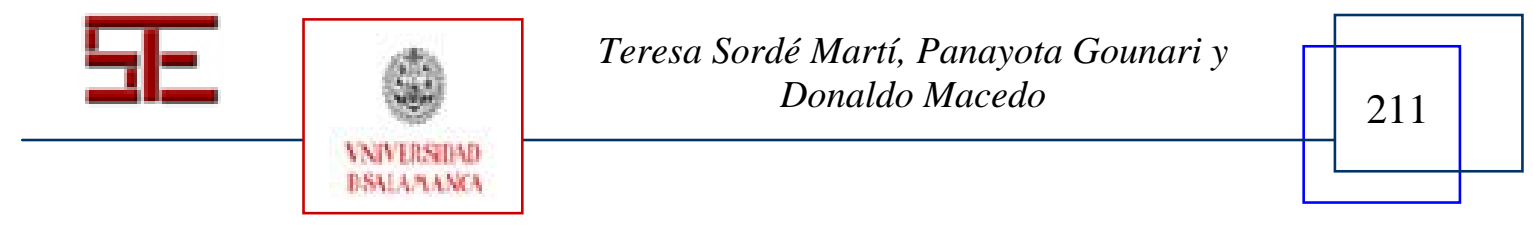


necesario estar abiertos a las propuestas que estas personas hagan, en lugar de cuestionarse y rechazarlas. El temor de que pase esto segundo limita muchísimo la aparición de nuevas iniciativas e implicaciones de personas del entorno próximo a las escuelas.

Dirigir esfuerzos a la creación de espacios de gestión y trabajo compartidos por personas de diferentes ámbitos tiene consecuencias muy positivas. Implicarse en un proyecto requiere no sólo compartir un mismo objetivo, sino también sentirse protagonista, comprobar que tu tarea es valorada y que se escucha tu voz. Hacer que se sientan protagonistas significa invitar a las familias a participar en lo que es importante dentro de las escuelas, como el fomento del aprendizaje, la lucha para superar el absentismo o la intervención en temas organizativos y de coordinación, y no sólo a la hora de organizar fiestas, confeccionar disfraces para carnaval o decorar la escuela. Una de las tareas en que más se implican las familias y en la que su aportación a la comunidad es más consistente es cuando entran a las aulas para apoyar al profesorado. En estos casos el alumnado se siente más atendido, y se incorporan a las aulas referentes culturales importantes.

Mediante el diálogo igualitario se incluyen las voces de todas las personas que forman parte de la escuela y, a partir de ahí, la escuela deja de ser una institución pensada desde y para la cultura mayoritaria para ser una institución multicultural construida por todas las personas y culturas, que participan en un plano de igualdad, sin priorizar unos valores por encima del resto. El diálogo igualitario se basa en la fuerza del argumento en lugar de hacerlo en la fuerza del poder de la persona que plantea este argumento. En este sentido, la aportación que hace un profesor o la directora de un centro tiene el mismo valor que la que puede hacer una abuela gitana o un padre sin estudios. Así se posibilita que toda persona aporte el conocimiento que ha adquirido a lo largo de su vida y su experiencia desarrollada en diferentes ámbitos, algunos más académicos y otros más prácticos.

Las aportaciones que las personas hacen mediante un diálogo igualitario no son privilegiadas por encima del resto, sino que todas se confrontan, se debaten y se valoran entre todos y todas las participantes, hasta llegar a acuerdos y consensos (Aubert et al., 2008). Con todo, la función de la figura del profesorado sufre algunas variaciones. Por un lado, debe abrirse a todas las aportaciones, iniciativas y contribuciones que se hagan, y valorar en un plano de igualdad y, por otro lado, también tiene la obligación de aportar sus conocimientos académicos y educativos. Sólo con un diálogo igualitario, una comunidad educativa formada por maestros, familiares y miembros de la comunidad podrá hacer realidad todos aquellos sueños definidos conjuntamente.

\section{5.- CONCLUSIÓN}

Nos gustaría finalizar insistiendo en que debemos tener el coraje y la integridad ética para denunciar cualquier y todos los intentos para deshumanizar activamente a los estu-

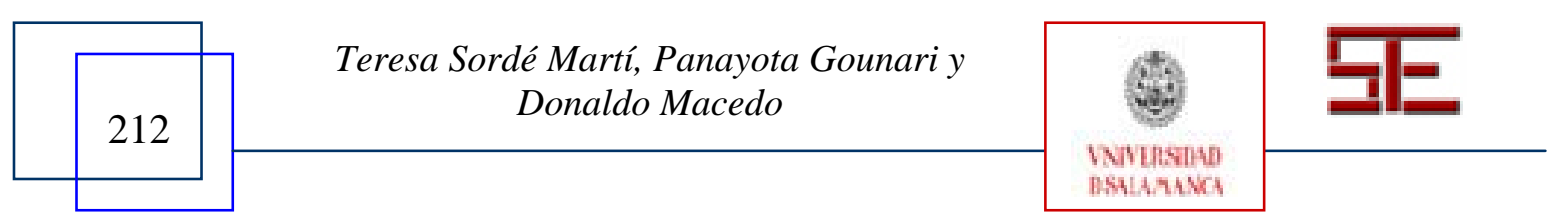




\begin{tabular}{c|c} 
Revista Electrónica Teoría de la Educación. \\
Educación y Cultura en la Sociedad de la Información.
\end{tabular}

diantes inmigrantes o gitanos a partir de los cuales nos ganamos la vida como profesores o trabajadores sociales. Hacer lo contrario es adoptar intencionadamente una forma de no jugar limpiamente con la alfabetización y la pobreza. Necesitamos tener el coraje y la integridad ética para decir que ningún ser humano es ilegal y menos extranjero o ajeno a la escuela o a la sociedad. La legalidad no se traduce siempre en moralidad. Cuando la esclavitud era legal, nunca ganó ningún principio moral.

Queremos reiterar la importancia de un diálogo desde abajo para hacer realidad la igualdad que forma parte de la pedagogía de la esperanza como también la tolerancia, el respeto y la solidaridad. Una pedagogía que rechaza la construcción social de imágenes que deshumanicen a los "otros”; una pedagogía que señale que, en nuestra construcción del otro, nos atamos íntimamente a ese otro; una pedagogía que nos enseñe que, deshumanizando al otro nos deshumanizamos a nosotros mismos. En pocas palabras, necesitamos una pedagogía de la esperanza que nos guíe hacia el camino crítico de la verdad, más que mitos y mentiras, hacia reclamar nuestra dignidad y nuestra humanidad. Una pedagogía de la esperanza nos encaminará hacia un mundo más armonioso y más humano, menos discriminatorio, menos deshumanizador y más justo. La pedagogía de la esperanza rechaza políticas de odio, intolerancia y división mientras celebra la diversidad dentro de la unidad. En otras palabras, una pedagogía de la esperanza señala, según Paulo Freire,

[el] camino a través del cual hombres y mujeres pueden concienciarse sobre su presencia en el mundo. La manera en que ellos actúan y piensan cuando desarrollan todas sus capacidades, teniendo en cuenta sus necesidades, pero también las necesidades y aspiraciones de otros ${ }^{7}$ (Freire y Betto, 1989, 32).

La creación de la alteridad no sólo fomenta más ignorancia por parte de los que están en el poder, sino que también fracasa en proveer al grupo dominante con las herramientas necesarias para tener empatía con el otro demonizado. El grupo dominante pierde su humanidad en su incapacidad de sentirse mal por discriminar a otros seres humanos. La capacidad del grupo dominante de demonizar y su incapacidad de tener empatía con el otro señalan el demonio inherente en aquellos que deshumanizan.

Mientras deshumanizamos a los extranjeros y a los llamados ilegales, también olvidamos que, según Carlos Fuentes, "ellos están recogiendo la comida que necesitamos sobre nuestras mesas, nos están sirviendo y cuidando de nuestros niños, están cortando nuestros setos y conduciendo nuestros autobuses y trayendo dignidad a las tareas indispensables, nunca "humildes", de la vida. Sin ellos, los precios se dispararían, la inflación se hincharía y la comida sería escasa”8 (Fuentes, 1999, 15).

En vez de deshumanizar ilegales, deberíamos aceptar el reto de Carlos Fuentes de imaginarnos los hijos de ilegales también como "los jóvenes profesores de sus propios y de otros, son los nuevos hombres de negocios expandiendo y diversificando los servicios y

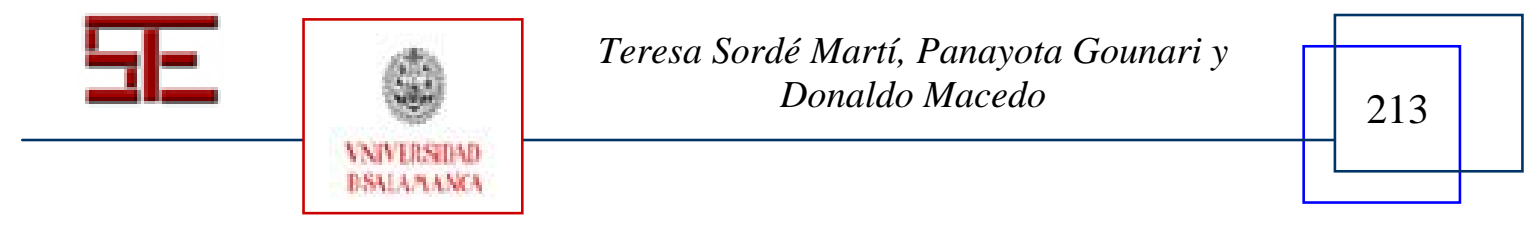


producción de los EEUU, son los nuevos doctores y abogados y arquitectos y biólogos y políticos, son los nuevos cantantes y actores y bailarines y directores de teatro y pintores y músicos enriqueciendo la cultura de los EEUU con contraste, diversidad y generosidad" (Fuentes, 1999, 15) ${ }^{9}$.

Imaginar la posibilidad humana prevista por Carlos Fuentes requiere tanto humanidad como humildad -una humildad que guíe y dé forma al acto de interpretar el mundo mientras se dé la coherencia necesaria a la interpretación del mundo un proceso a partir del cual podemos denunciar valientemente, con ira y agresivamente cualquier y todas las formas de desigualdad y discriminación conformadas por el racismo, de manera que podamos anunciar una nueva lectura del mundo desde abajo que, según Howard Zinn,conserve viva la memoria de la resistencia de las personas [que] sugieren un nuevo tipo de poder. Imaginad el pueblo [del mundo] unido por primera vez en un movimiento para el cambio fundamental. Imaginad el poder de la sociedad arrebatado desde corporaciones gigantes, militares y políticas que responden a intereses empresariales y militares.

Necesitaríamos reconstruir la economía para la eficiencia y la justicia. Empezaríamos en nuestros vecindarios, ciudades u lugares de trabajo. Se encontraría trabajo para todo el mundo. La sociedad se beneficiaría de la enorme energía, destreza y talento que ahora no se utiliza. Los básicos -comida, vivienda, cuidado de la salud, educación, transporte- serían accesibles para todos... inspirado [en] el pasado del pueblo, con su historia de resistencia, desobediencia civil contra la máquina militar, protestas contra el racismo, multiculturalismo e ira creciente contra inacabables guerras (Zinn, 2007, 212-213) ${ }^{10}$.

\section{6.- REFERENCIAS}

ANDRADE, M. (11/05/2009): Berlusconi advierte que no tolerará una "Italia multiétnica", El País. Disponible en:

http://www.elpais.com/articulo/internacional/Berlusconi/advierte/tolerara/Italia/m ultietnica/elpepuint/20090511elpepiint_5/Tes.

AUBERT, A.; DUQUE, E.; FISAS, M. y VALLS, R. (2004): Dialogar y transformar. Pedagogía crítica del siglo XXI. Barcelona, Graó.

AUBERT, A.; FLECHA, A.; GARCÍA, C.; FLECHA, R. y RACIONERO, S. (2008): Aprendizaje dialógico en la sociedad de la información. Barcelona, Hipatia Editorial.

BBC News (10/05/2009): Immigrants hurt in Greek violence. BBCNews, Disponible en: http://news.bbc.co.uk/go/pr/fr/-/2/hi/europe/8042409.stm.

CAHN, C. y CHIRICO, D. (1999): A Special Remedy: Roma and Schools for the Mentally Handicapped in the Czech Republic Country Reports Series. Budapest, European Roma Rights Center.

DANOVA, S. (27/04/2001): Desegregation of 'Romani Schools' A Condition for an Equal Start for Roma. Comunicación presentada en Desegregation of 'Romani Schools' A Condition for an Equal Start for Roma. Sofia, Bulgaria.

ECRE (2003): The Impact of Special Schools on the Roma in Central Europe. Brussels: ECRE.

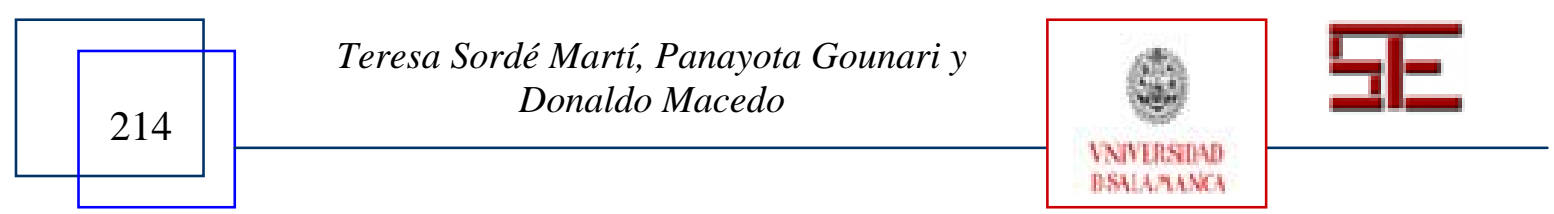


ELBOJ, C.; PUIGDELLÍVOL, I.; SOLER, M. y VALLS, R. (2002): Comunidades de aprendizaje. Transformar la educación. Barcelona: Graó.

EUROPEAN COURT OF HUMAN RIGHTS (2007): GRAND CHAMBER CASE OF D.H. AND OTHERS v. THE CZECH REPUBLIC. (Application $n^{\circ}$. 57325/00), Strasbourg [2007, November 13]. Disponible en: http://www.errc.org/db/02/D1/m000002D1.pdf.

FLECHA, R. (1990): La nueva desigualdad cultural. Barcelona, El Roure (2a edición publicada en 1994).

-- (2000): Sharing Words. Lanham, M.D, Rowman \& Littlefield.

FLECHA, R. et al., (2006-2011): INCLUD-ED. Strategies for inclusion and social cohesion from education. Proyecto integrado del VI Programa Marco de la Comisión Europea.

FREIRE, P. (1997): Pedagogy of the Heart. New York, The Continuum Publishing Company.

FREIRE, P., BETTO, F. (1989): Essa escola chamada vida. São Paulo, Editora Scipione.

FUENTES, C. (1999): Introduction. En Olmos, E. J.; Ybarra, L. y Monterrey, Manuel: Americanos: Latino life in the United States. Boston, MA, Little, Brown and Company.

GILROY, P. (2000): Against Race: Imagining Political Culture beyond the Color Line. Cambridge, MA, Harvard University Press, 2000.

GOLDBERG, T. (1993): Racist Culture: Philosophy and the Politics of Meaning. Oxford, Blackwell.

HABERMAS, J. (1984): The Theory of Communicative Action, vol. 1: Reasons and the Rationalization of Society. Boston, Beacon Press.

-- (1987): The Theory of Communicative Action, vol. 2: Lifeworld and System: A Critique of Functionalist reason. Boston, MA, Beacon Press.

HELSINKI WATCH REPORT (1992): Struggling for Ethnic Identity. Czechoslovakia's Endangered Gypsies. New York, Washington, Los Angeles, London, Human Rights Watch.

MIRGA, A. (2009): Hard Times and Hardening Attitudes: The Economic Downturn and the Rise of Violence against Roma. Witness Statement. Disponible en: http://www.osce.org/documents/odihr/2009/06/38091_en.pdf.

PARLAMENTO EUROPEO (2009): Resolución del Parlamento Europeo, de 2 de abril de 2009, sobre la educación de los hijos de los inmigrantes (2008/2328(INI)). Disponible en: http://www.europarl.europa.eu/sides/getDoc.do?pubRef=//EP//TEXT+TA+P6-TA-2009-0202+0+DOC+XML+V0//ES\&language=ES.

PRESTON, J. (17/11/2007): Immigration Quandary: A Mother Torn From Her Baby, New York Times. Disponible en: http://www.nytimes.com/2007/11/17/us/17citizen.html?_r=2\&ref=us\&pagewant ed=all.

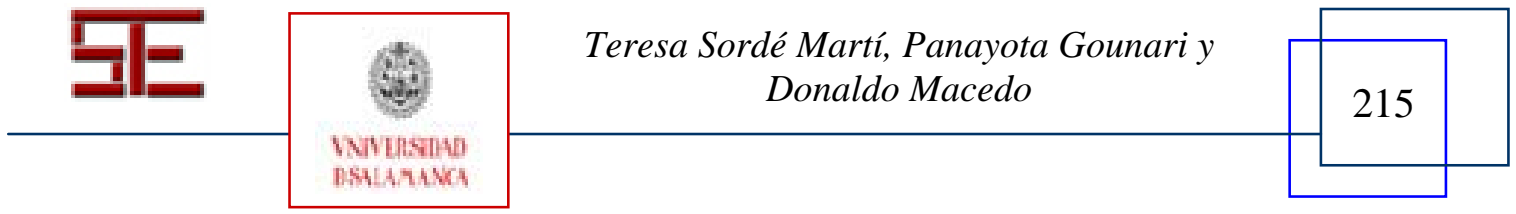


RIZZO, A. (28/03/2006): Berlusconi: Italy must not become 'multiethnic, multicultural country'. Nort County Times. Disponible en: http://www.nctimes.com/articles/2006/03/29/news/nation/16_05_463_28_06.txt.

ZINN, H. (2007): (Adapted by Rebecca Stefoff) A Young People's History of the United States. New York, Seven Stories Press.

\section{Notas}

${ }^{1}$ Citado en (Rizzo, 2006).

${ }^{2}$ Texto original: "If race is a conception, then racism is a condition; or more precisely, where race is a set of conceptions, racisms are sets of conditions".

${ }^{3}$ Texto original: "the dominant feature of racist expression".

${ }^{4}$ Texto original: "Racisms assume their particular characters, they are exacerbated, and they have different entailments and ramifications in relation to specific considerations of class constitution, gender, national identity, region and political structure".

${ }^{5}$ Texto original: "political interaction has been banished".

${ }^{6}$ Texto original: "Leaves unexplained the fact that racist expressions may at times define and promote rather that merely rationalize social arrangements and institutions” (p. 95).

7 Texto original: "[the] path through which men and women can become conscious about their presence in the world. The way they act and think when they develop all their capacities, taking into consideration their needs, but also the needs and aspirations of others".

${ }^{8}$ Texto original: "They are picking the food that we need on our tables, they are serving us our meals and taking care of our children, they are cutting our hedges and driving our buses and bringing dignity to the indispensable, never the 'lowly,' the indispensable tasks of life. Without them, prices would rockect, inflation would inflate, and food would be scarce".

${ }^{9}$ Texto original: "The young teachers of their own and others, they are the new businesspeople rapidly growing and diversifying U.S. services and production, they are the new doctors and lawyers and architects and biologists and politicians, they are the new singers and actors and dancers and stage directors and painters and musicians enriching U.S. culture with contrast, diversity, and generosity".

${ }^{10}$ Texto original: "Keeps alive the memory of people's resistance [that] suggests new kind of power. Imagine the [world] people united for the first time in a movement for fundamental change. Imagine society's power taken away from giant corporations, the military, and the politicians who answer to corporate and military interests. [...]We would need to rebuild the economy for efficiency and justice. We would start on our neighborhoods, cities, and workplaces. Work would be found for everyone. Society would benefit from the enormous energy, skill, and talent that is now unused. The basics — food, housing, health care, education, transportationwould be available to all... inspired [in] the people's past, with its history of resistance, civil disobedience against the military machine, protests against racism, multiculturalism, and growing anger against endless wars”.

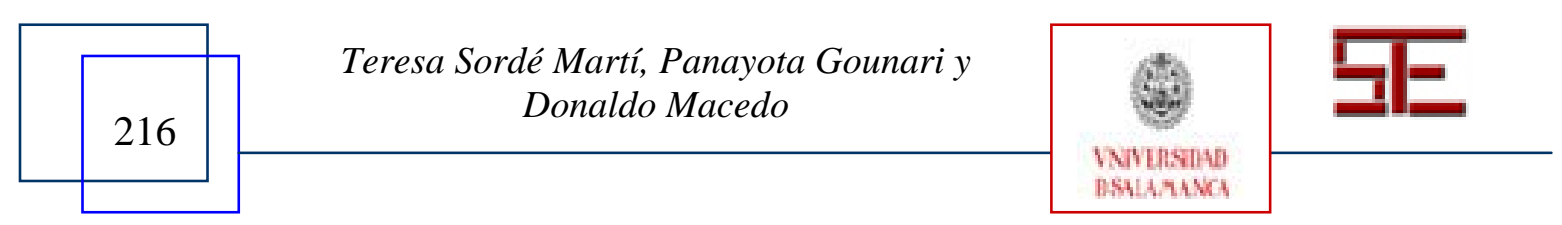




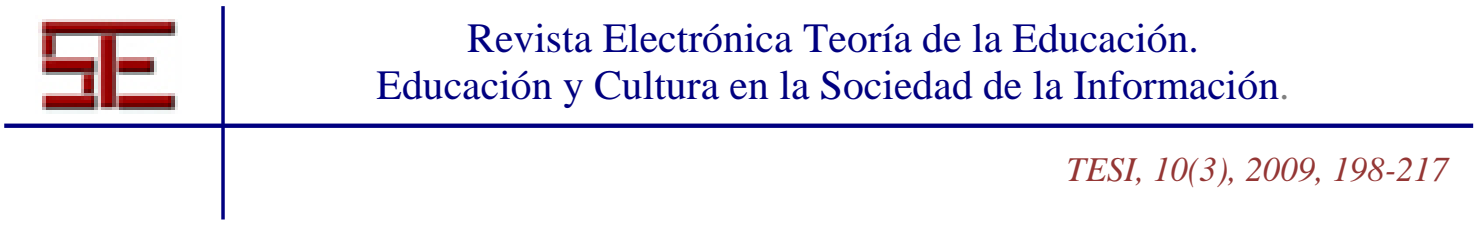

Para citar el presente artículo puede utilizar la siguiente referencia:

SORDÉ MARTÍ, T., GOUNARI, P. y MACEDO, D.: (2009): Avanza la crisis y el racismo: respuesta desde abajo, en FLECHA GARCÍA, R. y STEINBERG, S. (Coords.) Pedagogía Crítica del S. XXI [monográfico en línea]. Revista Electrónica Teoría de la Educación: Educación y Cultura en la Sociedad de la Información. Vol. 10, nº 3. Universidad de Salamanca, pp. 198-217. [Fecha de consulta: dd/mm/aaaa].

http://campus.usal.es/ revistas_trabajo/index.php/revistatesi/article/view/3970/3992 ISSN: 1138-9737

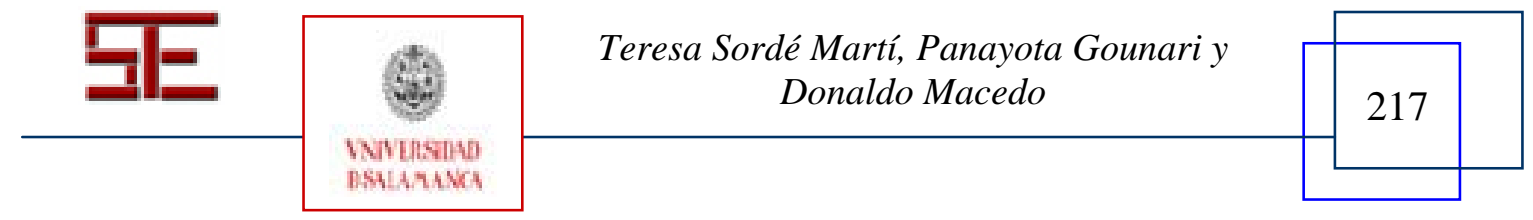

